\title{
RIDER OPTIMAL CONTROL IDENTIFICATION IN BICYCLING
}

\author{
A. L. Schwab* \\ P.D.L. de Lange \\ Laboratory for Engineering Mechanics \\ Delft University of Technology \\ Mekelweg 2, NL-2628 CD Delft, The Netherlands \\ e-mail: a.l.schwab@tudelft.nl
}

\author{
Jason K. Moore \\ Sports Biomechanics Lab \\ Mechanical and Aerospace Engineering \\ University of California, Davis \\ One Shields Avenue \\ Davis, CA 95616-5295, USA
}

\begin{abstract}
Rider control in bicycling is modeled by first adding the rider as a passive mechanism to the Whipple bicycle model. Next, for the rider control model a linear PID controller with and without delay is assumed, where the control inputs are the bicycle roll and steer angle with their higher derivatives, and the control output is the action-reaction steer torque applied by the rider at the handle bars. The experimental data is obtained from riding a bicycle on a narrow treadmill while applying an intermitted lateral perturbation by means of an impulse force applied at the seat post. The experiments are conducted in both the stable and the unstable forward speed range. After some filtering, a parametric control model is fitted to the data. Finally, the gains of this control model are used to identify the specific optimal control $L Q R$ cost function which the rider is using to control the bicycle on the treadmill at the various forward speeds.
\end{abstract}

\section{INTRODUCTION}

Balancing a bicycle in motion is an acquired skill which is poorly understood. Multibody dynamic models of the uncontrolled bicycles have provided fundamental insight into bicycle stability in relation to speed and geometry [1,2]. Further insight into human control is needed, e.g. to design bicycles, possibly with augmented control, minimizing risks of falling.

The research in human rider control in bicycles and motorcycles started in the seventies during the renewed interest in cybernetics $[3,4,5]$. Among the first were Van Lunteren \& Stassen [3] who used a stationary bicycle setup, mimicking normal bicycling, to investigate the influence of drugs and alcohol

*Address all correspondence to this author. on the performance of the rider. With the same setup they performed some system identification of the rider control at one fixed forward speed, where they adequately described the rider as a linear proportional integral derivative (PID) controller with delay. Rice \& Roland [4] measured rider control behavior after an initial lateral perturbation at various speeds on various bicycles and compared the results to computer simulations. Weir [5] used a computer model of a motorcycle rider combination to identify the transfer functions of the various control input-output relations, and concluded that steer torque to roll angle is the easiest way to balance a motorcycle in motion. After these pioneering studies, most attention has been directed to high-speed motorcycle rider control for reasons of traffic safety [6]. However, the act of balancing at low speed, as is the case for bicycles, has been given little attention. Only this decade, the research on low speed human rider control in bicycling was started again at TU Delft and UC Davis, by observing motions of various riderbicycle combinations while balancing a bicycle in motion on a large treadmill [7].

Currently there are two main modeling approaches on human rider control in bicycling. One builds on the well-developed linear aircraft pilot model as developed by McRuer et al. [8,9] and are transferred to the control of a bicycle. The other is more about intermittent control, where the rider has no action until a certain threshold and then performs an impulsive like action (e.g. Doyle [10]). Such systems are essentially nonlinear, and parallels can be drawn with the recent human postural balance research by Milton [11]. Here we have investigated the validity of the first type of models, that is, a linear controller with or without delay, and see from an optimal control perspective what the rider is optimizing. 
The outline of the paper is as follows. After this introduction the model of the bicycle-rider combination which is used in the system identification process is presented. Then the method of the applied system identification techniques are discussed. Next the measurements are briefly discussed after which the results of the system identification and the optimization strategy are presented. The paper ends with a discussion of the results and some conclusions.

\section{METHODS}

For the rider model we assume a linear PID controller with or without delay, where the control inputs are the bicycle roll and steer angle with their higher derivatives, and the control output is the steer torque. The rider is assumed to be rigidly attached to the rear frame. The experimental data is obtained from UC Davis [12], where experiments concerning rider control in bicycling are in progress. In these experiments, the bicycle is ridden on a narrow treadmill and intermittently laterally perturbed by an impulsive force at the seat post, see Figure 1. The experiments were done at three forward speeds: $3.2,4.3$ and $7.4 \mathrm{~m} / \mathrm{s}$, and by such covering both unstable and stable uncontrolled lateral motions. First a nonparametric final impulse response (FIR) model is derived, which served as a platform for subsequent parametric modeling. Next a linear PID parametric model is fitted to the non-parametric model using the steer angle signal. Finally the gains form this model are used in an linear quadratic regulator (LQR) optimal control scheme to see what the rider is optimizing.

\section{EXPERIMENTAL SETUP}

At UC Davis a measurement bicycle is constructed, which is equipped with a number of sensors to measure the state and rider input, see Figure 1. In addition, a perturbator mechanism is present, which is used to excite the system. These perturbations are applied by laterally pulling a rope with a force sensor in series, which is attached on the seat post. The measurement bicycle has the following characteristics: the upper body lean is constrained by rigidly fixing the upper body with a harness to the bicycle frame in order to mimic the rigid rider bicycle model (Whipple model) as best as possible, the bicycle is electrically driven, so the rider does not need to exert pedaling power and thus eliminates the need for lower limb movement, and the knees are fixed to the bicycle frame, which prevents the lateral knee movement which was observed in [7].

The experiments are performed on a narrow treadmill were the task is to keep the bicycle in balance. The treadmill proved to be very suitable for the perturbation experiments, since it is easy to perturb a stationary positioned bicycle by pulling the rope. A downside of this environment is the rather narrow track, resulting in a stressful and unnatural overly concentrated way of bi-

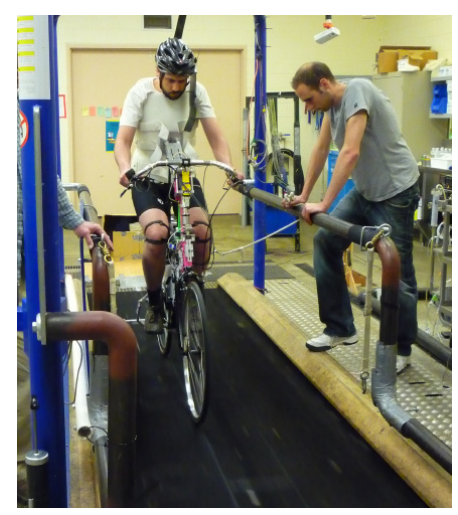

FIGURE 1. Experimental setup at UC Davis of an instrumented and actuated bicycle riding on a narrow treadmill. The lateral perturbation is an impulsive pulling force at the seat post.
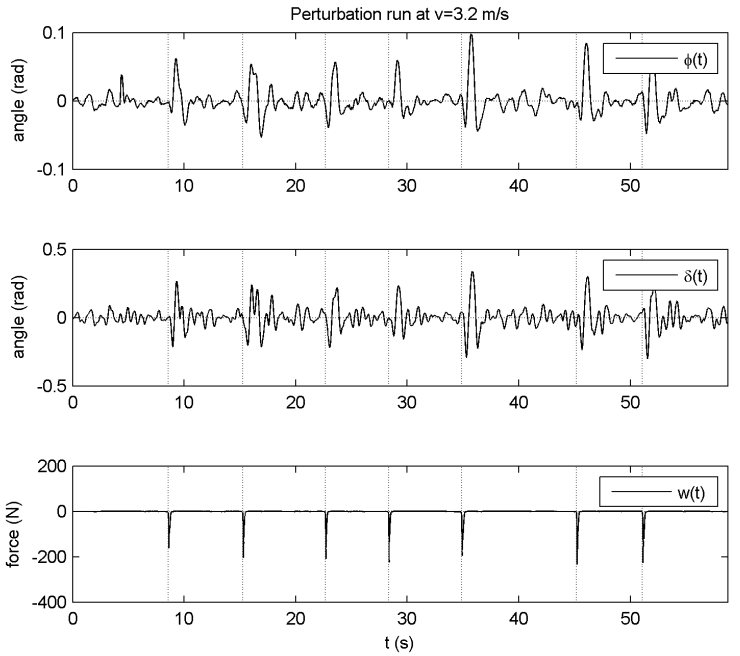

FIGURE 2. Measurements of the roll angle $\phi$ (top), steering angle $\delta$ (middle) and disturbance $w$ (bottom) for a forward velocity of $3.2 \mathrm{~m} / \mathrm{s}$.

cycling. The treadmill perturbation experiments were performed at forward velocities of 3.2, 4.3 and $7.4 \mathrm{~m} / \mathrm{s}$ with a measurement time of 60 to 90 seconds per run, each of which is repeated a number of times. The measured data during the experiment are: the forward velocity $v$, the rear frame roll angle $\phi$ and roll rate $\dot{\phi}$, the steer angle $\delta$ and steer rate $\dot{\delta}$, the disturbance force applied at the seat post $w$, and the steering torque $T_{\delta}$. Unfortunately the latter showed large errors and was not used in the identification process. Figure 2 shows a typical measurement of the roll angle, steering angle and input force. 


\section{SYSTEM MODEL}

The total system is a combination of a bicycle model and a rider model. For the bicycle the Whipple [13] rigid rider model will be used. Whereas, the rider control will be modeled as a linear feedback control system with inherent neuromuscular lag and time delays.

The Whipple bicycle model [13], which recently has been benchmarked [1], consists of four rigid bodies connected by revolute joints. The contact between the knife-edged wheels and the flat level surface is modelled by holonomic constraints in the normal direction, prescribing the wheels to touch the surface, and by non-holonomic constraints in the longitudinal and lateral directions, prescribing zero longitudinal and lateral slips. In this original model, it is assumed that the rider is rigidly attached to the rear frame and has no hands on the handlebar. The resulting non-holonomic mechanical model has three velocity degrees of freedom: forward speed $v$, roll rate $\dot{\phi}$ and steering rate $\dot{\delta}$. The lateral motions can be described by the linearized equations of motion for small perturbations about the upright steady forward speed $v$, and have the structure,

$$
\mathbf{M} \ddot{\mathbf{q}}+v \mathbf{C}_{1} \dot{\mathbf{q}}+\left[g \mathbf{K}_{0}+v^{2} \mathbf{K}_{2}\right] \mathbf{q}=\mathbf{f},
$$

where the time-varying variables are $\mathbf{q}=[\phi, \delta]^{\mathrm{T}}$ and the roll and steering torques are $\mathbf{f}=\left[T_{\phi}, T_{\delta}\right]^{\mathrm{T}}$. The entries in the constant coefficient mass matrix $\mathbf{M}$, damping-like matrix $\mathbf{C}_{1}$ (there is no real damping in the system), and constant and velocity dependent stiffness matrices $\mathbf{K}_{0}$ and $\mathbf{K}_{2}$ can be calculated from a nonminimal set of 25 bicycle parameters as described in [1]. For the instrumented bicycle from Figure 1, where the 25 bicycle parameters where measured using the methods described in [14], the resulting matrices are:

$$
\begin{gathered}
\mathbf{M}_{\mathbf{0}}=\left[\begin{array}{rr}
131.5085 & 2.6812 \\
2.6812 & 0.2495
\end{array}\right], \mathbf{C}_{\mathbf{1}}=\left[\begin{array}{cr}
0 & 42.748 \\
-0.31806 & 1.6022
\end{array}\right], \\
\mathbf{K}_{\mathbf{0}}=\left[\begin{array}{ll}
-116.19 & -2.7633 \\
-2.7633 & -0.94874
\end{array}\right], \mathbf{K}_{\mathbf{2}}=\left[\begin{array}{ll}
0 & 102.02 \\
0 & 2.5001
\end{array}\right] .
\end{gathered}
$$

Because the forward speed is a parameter, the stability of the lateral motions expressed by the eigenvalues from the linearized equations of motion (1), also show this forward speed dependency. These eigenvalues of the uncontrolled instrumented bicycle, in the forward speed range of $0 \mathrm{~m} / \mathrm{s}<v<10 \mathrm{~m} / \mathrm{s}$, are presented in Figure 3. In principle, there are up to four eigenmodes, where oscillatory eigenmodes come in pairs. Two are significant and are traditionally called the capsize mode and the weave mode, see Figure 3. The capsize mode corresponds to a real eigenvalue with an eigenvector dominated by lean: when unstable, the bicycle follows a spiralling path with increasing curvature until it falls. The weave mode is an oscillatory motion in which the bicycle sways about the heading direction. The third remaining eigenmode is the overall stable castering mode, like in a trailing caster wheel, which corresponds to a large negative real eigenvalue with an eigenvector dominated by steering. In the unstable region $\left(v<v_{\text {weave }}\right)$ there is clearly need for rider control.

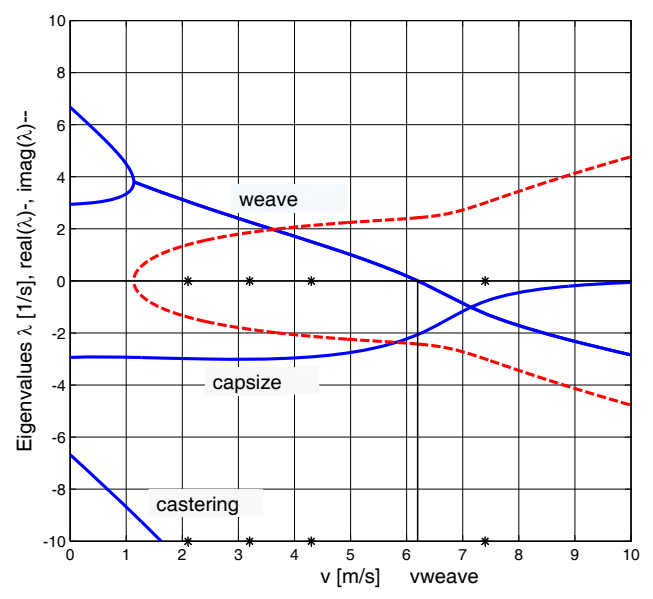

FIGURE 3. Eigenvalues for the uncontrolled instrumented bicycle from figure 1 in the forward speed range $0<v<10 \mathrm{~m} / \mathrm{s}$, solid lines are the real values and dashed lines are the imaginary values. The speed where the weave motion becomes stable is $v_{\text {weave }} \approx 6.2 \mathrm{~m} / \mathrm{s}$. The capsize mode becomes mildly unstable at $v_{\text {capsize }} \approx 10.8 \mathrm{~m} / \mathrm{s}$, which is not shown in the figure. Forward speeds used in the experiments are donated by an $*$.

The rider control model is assumed to be a linear feedback system in series with neuromuscular lag and time delay. The linear feedback system is usually written as $\mathbf{u}(s)=\mathbf{K}(s) \mathbf{y}(s)$ with the control input $\mathbf{y}$, control output $\mathbf{u}$, and feedback gains $\mathbf{K}$. In our model the rider control input is assumed to be the bicycle roll and steer angle, $\mathbf{y}=[\phi, \delta]^{T}$, and for the rider control output we assume steer torque only, $\mathbf{u}=\left[T_{\delta}, \mathrm{u}\right]$. This rider control output then acts as input to the bicycle model, $\mathbf{f}=[0,1]^{T} \mathbf{u}$, and by such closes the control loop. Next we introduce a number of sensory feedback gains, which act linearly on the bicycle configuration output. We assume the rider to be capable of sensing and applying proportional, integrative, first and second order derivative action. These assumptions may be modeled mathematically according to,

$$
\begin{aligned}
& K_{\phi}(s)=k_{\phi p}+k_{\phi i} s^{-1}+k_{\phi d} s+k_{\phi d d} s^{2} \\
& K_{\delta}(s)=k_{\delta p}+k_{\delta i} s^{-1}+k_{\delta d} s+k_{\delta d d} s^{2}
\end{aligned}
$$

with roll angle feedback $K_{\phi}$ and steer angle feedback $K_{\delta}$, and $s$ 


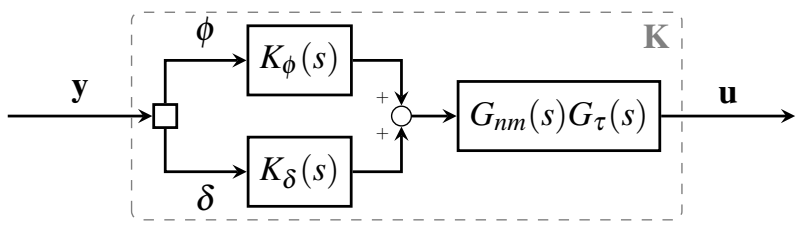

FIGURE 4. Block diagram of the inner control structure of $\mathbf{K}$, with roll and steering angle feedback gains $K_{\phi}$ and $K_{\delta}$, timedelay $G_{\tau}$, neuromuscular lag $G_{n m}$, input $\mathbf{y}=[\phi, \delta]^{T}$ and output $\mathbf{u}=\left[T_{\delta, \mathrm{u}}\right]^{T}$.

the Laplace argument. The gains $k$ with subscript $p, i, d$ and $d d$ indicate proportional, integral, first and second order derivative gains respectively.

According to McRuer and Jex [15], the human controller is inherently limited by neuromuscular lag and time delays. Here the neuromuscular dynamics is modeled just like in the shoulder muscle model from [16,17], which yields, $G_{n m}(s)=$ $\omega_{c}^{2} /\left(s^{2}+2 \zeta \omega_{c}+\omega_{c}^{2}\right)$ with cuttof frequency $\omega_{c}=2.17 \cdot 2 \pi \mathrm{rad} / \mathrm{s}$ and damping coefficient $\zeta=\sqrt{2}$ (Figure 4). This system acts as a critically damped second order filter with a cuttoff frequency equal to $\omega_{0}$. Next the transport delays result in an effective time delay, which is modeled as a single delay, with transfer function $G_{\tau}(s)=e^{-\tau_{d} s}$. For the rider model we initially used a time delay of $\tau_{d}=0.03$ seconds.

Finally the human limitations and the linear feedback model are combined to form a rider control model according to, $\mathbf{K}(s)=$ $G_{n m}(s) G_{\tau}(s)\left[K_{\phi}(s), K_{\delta}(s)\right]^{T}$, which is presented as a block diagram in Figure 4. Since the lateral dynamics of the bicycle is strongly forward speed dependent, it is expected that the feedback gains will also show this forward speed dependency.

\section{IDENTIFIED RIDER MODEL}

The rider control system identification is done in three steps. First, a nonparametric Finite Impulse Response (FIR) model [18] is fitted to the raw data. Next, the FIR model is used to obtain a noise model. Finally, a parametric model according to (3) is used, which is optimized by using parameter reduction techniques. The analysis is performed for a number of forward speeds, resulting in a set of parametric models. Finally, the gains of this control model are used to identify the specific LQR cost function which the rider is using to control the bicycle at the various forward speeds.

The parametric model (3) has in principal eight feedback gains. However, the number of parameters can be reduced while still maintaining a good fit, expressed by the variance accounted for (VAF). The reduction technique is based on parameter selection with the help of the parameter sensitivity on the VAF determined by the parameter covariances [18]. The results of this process, the identified control feedback gains of the rider model, are shown in Table 1, where zero time delays are used because of in-

\begin{tabular}{rrrrrrr}
\hline $\mathrm{v}$ & $k_{\phi d}$ & $k_{\phi p}$ & $k_{\delta d}$ & $k_{\delta p}$ & $k_{\delta i}$ & $\mathrm{VAF}$ \\
$\frac{\mathrm{m}}{\mathrm{s}}$ & $\frac{\mathrm{Nms}}{\mathrm{rad}}$ & $\frac{\mathrm{Nm}}{\mathrm{rad}}$ & $\frac{\mathrm{Nms}}{\mathrm{rad}}$ & $\frac{\mathrm{Nm}}{\mathrm{rad}}$ & $\frac{\mathrm{Nm}}{\mathrm{s} \mathrm{rad}}$ & $\%$ \\
\hline 3.2 & 32.99 & 36.81 & -3.25 & 0 & 89.45 & 94.24 \\
& 78.51 & 26.95 & -2.08 & -19.91 & 54.40 & \\
\hline 4.3 & 33.79 & 29.58 & -2.74 & 0 & 195.40 & 98.75 \\
& 34.09 & 100.51 & -2.27 & -20.99 & 162.60 & \\
7.4 & 41.56 & 59.20 & -10.56 & 0 & 816.11 & 95.06 \\
& 46.48 & 139.79 & -2.83 & -27.57 & 804.39 & \\
\hline
\end{tabular}

TABLE 1. Results of the reduced parameter rider control model, with controller gains $\mathbf{K}$ (3), forward velocity $v$, no time delay $\tau_{d}=0$, and variance acounted for (VAF). The grey marked rows indicate the gains obtained from the optimal control approach, where the optimal criterium weights $\mathbf{Q}$ and $\mathbf{R}$ are presented in Table 2 .

\begin{tabular}{rrrrrrr}
\hline & \multicolumn{9}{c}{$Q$} & & $R$ \\
\cline { 2 - 7 } $\mathrm{v}$ & $\hat{\dot{\phi}}$ & $\hat{\phi}$ & $\hat{\dot{\delta}}$ & $\hat{\delta}$ & $\hat{\psi}$ & $\hat{\tau}_{\delta}$ \\
$\mathrm{m} / \mathrm{s}$ & $\mathrm{rad} / \mathrm{s}$ & $\mathrm{rad}$ & $\mathrm{rad} / \mathrm{s}$ & $\mathrm{rad}$ & $\mathrm{rad}$ & $\mathrm{Nm}$ \\
\hline 3.2 & $\infty$ & $\infty$ & $\infty$ & $\infty$ & 0.0547 & 1 \\
4.3 & $\infty$ & $\infty$ & $\infty$ & $\infty$ & 0.0246 & 1 \\
7.4 & $\infty$ & $\infty$ & $\infty$ & $\infty$ & 0.0086 & 1 \\
\hline \hline
\end{tabular}

TABLE 2. Optimal control weights for the state vector, $\mathbf{Q}$, and for the control effort $\mathbf{R}$, where a value $>1 e 6$ is donated by $\infty$. The weights are normalized with respect to the control effort $\tau_{\delta}$.

stability problems. The use of roll angle and roll angle rate represent vestibular and/or visual feedback, and the use of steer angle rate represents proprioceptive feedback. The signs of the gains on the roll angle and roll rate clearly show that the necessary steer-into-the-fall balance mechanism [2] for stabilizing the lateral motions is used by the rider. That is, a roll or roll rate to the right results in a control steer torque to the right. The feedback of the integral of the steer angle can be explained by the need for the rider to stay on the the narrow treadmill. Here the rider is controlling the heading of the bicycle within small bounds and the heading, $\psi$, is mainly determined by the integral of the steer angle, $\psi=\int(v \boldsymbol{\delta}+c \dot{\boldsymbol{\delta}}) / w \cos \left(\boldsymbol{\lambda}_{s}\right) d t \approx v / w \cos \left(\boldsymbol{\lambda}_{s}\right) \int \delta d t$, with the wheelbase $w$, the trail $c$ and the head angle $\lambda_{s}$. All feedback gains show a forward speed dependency, the most profound in the integral steering feedback, which seems to be quadratic in the forward speed.

Finally, we like to address the question: if the rider is operating like an optimizer then what is the rider optimizing? To an- 
swer this question we formulate the standard LQR optimal control problem [19] with the performance index (or cost function) for the control task

$$
J=\int\left(\mathbf{x}^{T} \mathbf{Q} \mathbf{x}+\mathbf{u}^{T} \mathbf{R} \mathbf{u}\right) \mathrm{dt},
$$

with the state vector $\mathbf{x}=\left(\dot{\phi}, \dot{\delta}, \phi, \delta, \int \delta\right)$ and the controller output $\mathbf{u}=\left(\tau_{\delta}\right)$, and the weight factors $\mathbf{Q}$ on the state and $\mathbf{R}$ on the control effort. The optimal feedback gains for a linear feedback controller are found by minimizing the performance index $J$, which is the well known Riccati solution (LQR model). Here, we work the other way around, given the feedback gains from the parameter identification process, what are the weight factors? We solve this by a nonlinear search on $J$. For the weights we assume all off-diagonal terms in $\mathbf{Q}$ and $\mathbf{R}$ zero, and the remaining unknown diagonal terms as $1 / \hat{x}_{i}^{2}$, where $\hat{x}_{i}$ is the maximal allowable value of the ith element of the state vector [19]. For the control effort weights $\mathbf{R}$, here a scalar, we write $R=1 / \hat{\tau}_{\delta}^{2}$, where $\hat{\tau}_{\delta}$ is identified as the maximum steer torque. The results of this process are shown in Table 2. Clearly, the rider is not optimizing with respect to the roll or steer angle and rate, indicated by $\infty$ values. When we normalize the weights with respect to the control effort, that is normalize with respect to the maximum steer torque $\tau_{\delta}$, we see that the rider has to pay more and more attention to the heading with increasing speed, which makes sense for the task of riding on a narrow treadmill.

\section{CONCLUSIONS}

The conclusions from these experiments and data analysis are that, if we assume that the rider is using a linear feedback control system, then he only uses the roll angle, roll rate, steer angle rate and integral of the steer angle (is heading) for feedback control. Next, if we assume that this linear control model is correct and if the rider is seen as an optimizer then in the task of riding on a treadmill he optimizes his control with respect to the heading angle only.

\section{ACKNOWLEDGEMENT}

We like to thank Mont Hubbard for hosting Peter de Lange at UC Davis and giving him the opportunity to join in on the measurement program. Thanks to Andy Ruina for the idea of trying to figure out what the rider is optimizing.

\section{REFERENCES}

[1] J. P. Meijaard, Jim M. Papadopoulos, Andy Ruina, and A. L. Schwab. Linearized dynamics equations for the balance and steer of a bicycle: a benchmark and review. Proceedings of the Royal Society A, 463:1955-1982, 2007.
[2] J. D. G. Kooijman, J. P. Meijaard, Jim P. Papadopoulos, Andy Ruina, and A. L. Schwab. A bicycle can be self-stable without gyroscopic or caster effects. Science, 332:339-342, April 15, 2011.

[3] A. van Lunteren and H. G. Stassen. On the variance of the bicycle rider's behavior. In Proceedings of the 6th Annual Conference on Manual Control, April 1970.

[4] Roy S. Rice and R. Douglas Roland. An evaluation of the perfomance and handling qualities of bicycles. Technical report, Cornell Aero. Lab. Rept. VJ-2888-K, April 1970. (available at: http://bicycle.tudelft.nl/schwab/Bicycle/calspan/).

[5] D. H. Weir. Motorcycle Handling Dynamics and Rider Control and the Effect of Design Configuration on Response and Performance. PhD thesis, University of California, LA, 1972.

[6] A. A. Popov, S. Rowell, and J. P. Meijaard. A review on motorcycle and rider modelling for steering control. Vehicle System Dynamics, 48:775-792, 2010.

[7] Jason K. Moore, J. D. G. Kooijman, A. L. Schwab, and Mont Hubbard. Rider motion identification during normal bicycling by means of principal component analysis. Multibody System Dynamics, 25(2):225-244, 2011.

[8] Duane T. McRuer, Dunstan Graham, and Ezra S. Krendel. Manual control of single-loop systems: Part i. Journal of the Franklin Institute, 283(1):1 - 29, 1967.

[9] Duane T. McRuer, Dunstan Graham, and Ezra S. Krendel. Manual control of single-loop systems: Part ii. Journal of the Franklin Institute, 283(2):145 - 168, 1967.

[10] A. J. R. Doyle. The skill of bicycle riding. $\mathrm{PhD}$ thesis, University of Sheffield, UK, 1987. (available at: http://bicycle.tudelft.nl/schwab/Bicycle/doyle1987skill.pdf).

[11] J. Milton, J.L. Cabrera, T. Ohira, S. Tajima, Y. Tonosaki, C.W. Eurich, and S.A. Campbell. The time-delayed inverted pendulum: Implications for human balance control. Chaos: An Interdisciplinary Journal of Nonlinear Science, 19:026110, 2009.

[12] http://biosport.ucdavis.edu/researchprojects/bicycle/instrumented-bicycle.

[13] F. J. W. Whipple. The stability of the motion of a bicycle. Quarterly Journal of Pure and Applied Mathematics, 30:312-348, 1899.

[14] J. D. G. Kooijman, A. L. Schwab, and J. P. Meijaard. Experimental validation of a model of an uncontrolled bicycle. Multibody System Dynamics, 19:115-132, 2008.

[15] D.T. McRuer and H.R. Jex. A review of quasi-linear pilot models. Human Factors in Electronics, IEEE Transactions on, HFE-8(3):231-249, Sept. 1967.

[16] E. de Vlugt, A.C. Schouten, and F.C.T. van der Helm. Closed-loop multivariable system identification for the characterization of the dynamic arm compliance using continuous force disturbances: a model study. Journal of neu- 
roscience methods, 122(2):123-140, 2003.

[17] R. Happee, E. De Vlugt, and A.C. Schouten. Posture maintenance of the human upper extremity; identification of intrinsic and reflex based contributions. SAE International Journal of Passenger Cars-Mechanical Systems, 1(1):1125, 2009.

[18] L. Ljung. System identification: theory for the user, volume 11. Prentice-Hall Englewood Cliffs, NJ, 1987.

[19] A.E. Bryson and Y.C. Ho. Applied optimal control. Wiley New York, 1975. 